\title{
Flow Characteristics and Control of Vertical Upward Gas-Liquid Two-Phase Flow through a Sudden Contraction Pipe*
}

\author{
Alexandros VOUTSINAS ${ }^{* *}$, Toshihiko SHAKOUCHI ${ }^{* *}$, Junichi TAKAMURA**, \\ Koichi TSUJIMOTO** and Toshitake ANDO**
}

\begin{abstract}
The flow characteristics of upward gas-liquid two-phase flow through a vertical sudden contraction pipe is an area that has been less studied experimentally and numerically and that is still little known ${ }^{(1)-(3)}$. The drag and its reduction for an upward bubbly two-phase flow in a sudden contraction pipe are investigated. Vortices are generated just prior to and after contraction and they significantly affect the flow characteristics. A simple and economical method to control and smoothen the flow is proposed by mounting a small obstacle (ring) upstream the contraction. The effects of ring position, Reynolds number and volumetric gas flow rate are examined and positive results were seen in all flow conditions. Moreover, the pressure fluctuation phenomenon and its control downstream the contraction was considered.
\end{abstract}

Key Words: Gas-Liquid Two-Phase Flow, Sudden Contraction Pipe, Flow Control, Drag Reduction, Fluctuating Flow

\section{Introduction}

Two-phase flow that passes through pipes is often seen in boilers, heat exchange devices, nuclear reactors and other hydraulic machines. Many studies concerning phase change and the flow regime characteristics of twophase flow through straight pipes have been conducted and a great deal of knowledge has been gathered ${ }^{(1)-(4)}$.

However, few studies have focused on two-phase flow characteristics in a vertical upward sudden contraction pipe and a few are mentioned in the bibliography ${ }^{(2),(8),(9)}$. Because of the contraction area, vortices are generated just prior to and after the contraction, significantly affecting the flow characteristics and pattern. Moreover, due to gasexistence (void fraction) the flow becomes even more unstable compared to single-phase flow.

In this study, flow characteristics of single and twophase flow through a vertical upward sudden contraction pipe and the fluctuating flow downstream the contraction are studied ${ }^{(5)}$. By inserting an obstacle (ring) before the contraction, the flow is detached and introduced more smoothly to the contraction area, which results in the suppression of the vortex generation downstream. This leads

* Received 9th March, 2006 (No. 06-4043)

** Graduate School of Engineering, Mie University, 1577 Kurimamachiya-cho, Tsu-shi, Mie 514-8507, Japan.

E-mail: shako@mach.mie-u.ac.jp to drag reduction since the pressure loss and flow stability due to pressure fluctuations are reduced. Experimental, visual and numerical works were conducted for a variety of parameters such as Re number, gas flow rate and contraction area ratio.

\section{Nomenclature}

$A$ : Sudden contraction area ratio

$d_{1}, d_{2}:$ Inner diameters

$D_{\mathrm{R}}:$ Drag reduction ratio

$f:$ Frequency

$H_{\mathrm{h}}:$ Elevation head

$L_{\mathrm{r}}:$ Ring position

$Q_{\mathrm{a}}:$ Air flow rate

$R e:$ Reynolds number

$U_{\mathrm{m}}:$ Water mean velocity

$C_{\mathrm{p}}:$ Pressure coefficient

$d_{\mathrm{bm}}:$ Bubble mean diameter

$f_{k}:$ Interfacial forces

$H$ : Step height

$h$ : Ring height

$p$ : Pressure

$Q_{\mathrm{w}}$ : Water flow rate

$T$ : Time

$\Delta p:$ Pressure drop

$\alpha_{\mathrm{v}}$ : Volumetric gas flow ratio 
$\varepsilon$ : Pressure gradient by experiment

$\varepsilon_{1}:$ Pressure gradient in Eq. (5)

$\lambda$ : Interfacial friction coefficient

$\lambda_{\kappa}:$ Friction factor

$v:$ Water viscosity

$\rho:$ Density

$\tau_{\kappa}:$ Viscous stress

\section{Subscript}

$$
\begin{aligned}
\mathrm{a} & : \text { Air } \\
\mathrm{b} & : \text { Bubble } \\
\mathrm{h} & : \text { Head } \\
\mathrm{g} & : \text { Gas } \\
\mathrm{l} & : \text { Liquid } \\
\mathrm{m} & : \text { Mean } \\
\mathrm{t} & : \text { Two-phase } \\
\mathrm{w} & : \text { Water }
\end{aligned}
$$

\section{Experimental Set-Up and Procedure}

Figure 1 shows the experimental set-up. The pump (2) sends the required amount of water through the water flow meter (4) into the test section which has a total length of $L=2450 \mathrm{~mm}$ up to the test section. The gas (air) needed is given by an air-compressor (12) after passing through the air dryer (13) and the air flow meter (4a).

For the creation of bubbles, a bubble generator made of porous fine ceramics was used and the volumetric flow rate ratio varied from $\alpha_{\mathrm{v}}=Q_{\mathrm{a}} /\left(Q_{\mathrm{a}}+Q_{\mathrm{w}}\right)=0 \sim 10 \%$. $Q_{\mathrm{a}}$ and $Q_{\mathrm{w}}$ are the volumetric flow rate of air and water, respectively. The water temperature was maintained at constant of 20 degrees Celsius and the mean bubble diameter at distance $2 d_{1}$ upstream the contraction step was found to be approximately $d_{\mathrm{bm}}=2.5 \mathrm{~mm}$ for $R e=1 \times 10^{4}$ and $\alpha_{\mathrm{v}}=10 \%$.

Figure 2 illustrates the sudden contraction area. The large pipe has an inner diameter $d_{1}=70 \mathrm{~mm}$ and the smaller pipes are $d_{2}=30,40 \mathrm{~mm}$ (Area ratio $A=0.18,0.33$ ) respectively. On the pipe surface prior to and after the contraction step, pressure taps $0.8 \mathrm{~mm}$ in diameter were drilled. There are 10 pressure holes before the contraction, with $10 \mathrm{~mm}$ distance between each and 23 holes after. As a reference point, a pressure hole at distance $2 d_{1}$ upstream the contraction step, where the flow was considered to be stable, was chosen.

In order to control the flow, an obstacle (ring) with a height of $h=0.4 H$ [where $H=\left(d_{1}-d_{2}\right) / 2$, step height] is mounted at various distances of $L_{\mathrm{r}}=(1 \sim 3) H$. However, in the present study only the results for $L_{\mathrm{r}}=1-2 \mathrm{H}$ are shown since in all flow conditions the ring position at $3 H$ had the worst results.

A water column manometer was used to measure pressure loss and distribution, while for the visualization experiments a high-speed camera, a video camera and a digital camera were used. The high-speed camera was

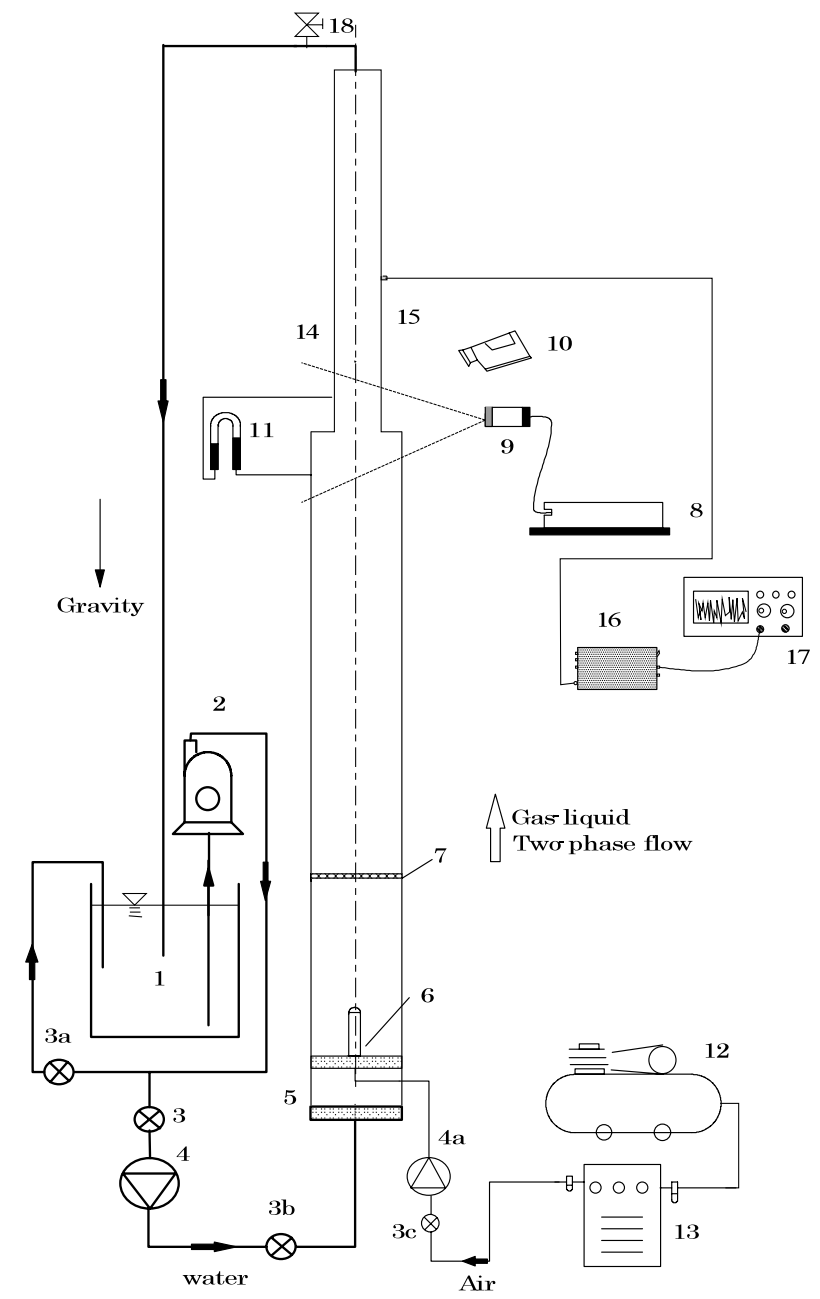

Fig. 1 Schematic diagram of experimental set-up
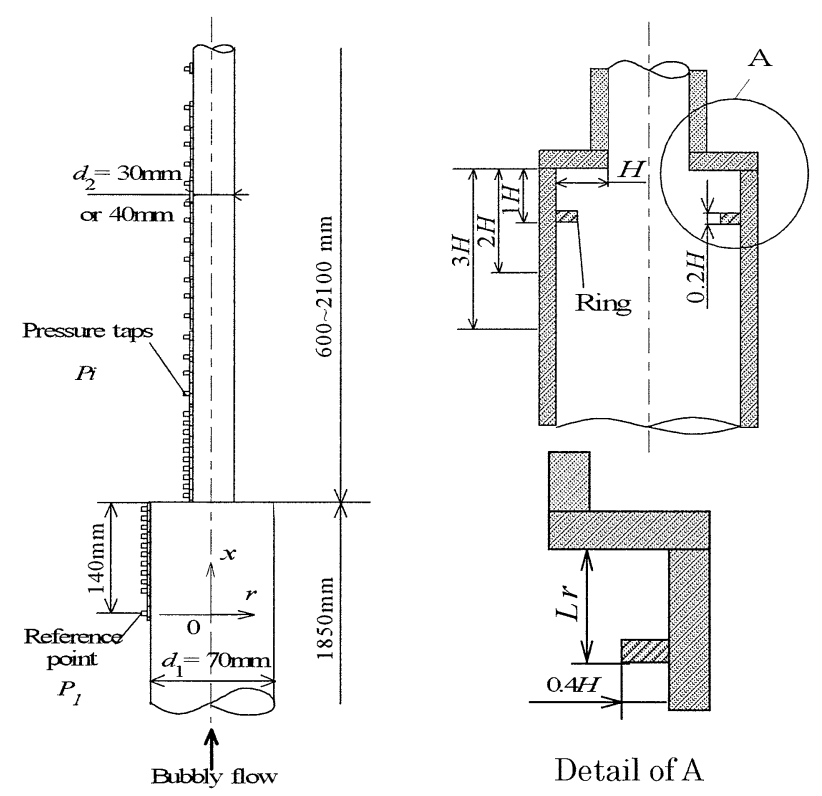

Detail of A

Fig. 2 Test section (Sudden contraction pipe) 


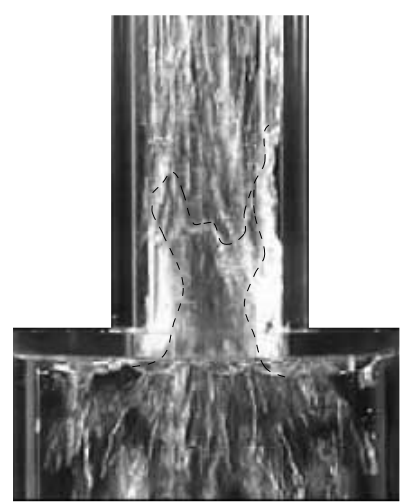

(a) Flow visualization

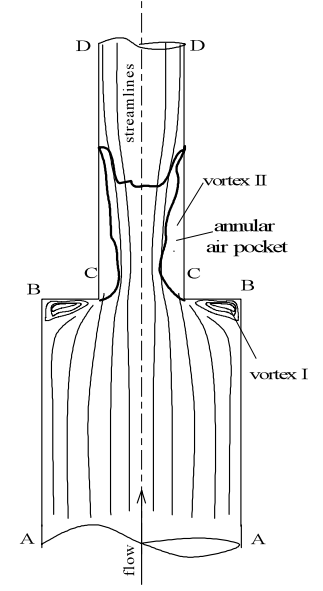

(b) Schematic flow diagram

Fig. 3 Flow pattern without ring $\left(R e=2 \times 10^{4}, \alpha_{\mathrm{v}}=5 \%, A=0.18\right)$

set at a shutter speed of $1 / 4000 \mathrm{sec}$ and a frame rate of $240 \mathrm{fps}$. For the visualization and flow analysis of singlephase (water) flow, styrene beads with 1.02 density and $0.03 \mathrm{~mm}$ diameter were used as tracers. In the case of two-phase flow, the bubbles movement was observed. A five $\mathrm{mm}$ slit of light from halogen lamps were used to illuminate the flow passage. The test section, in which the visual observations were made, was immersed in a transparent container filled with water in order to hold off the distortion that occurs due to pipe-wall curvature.

Experiments were conducted with Reynolds number variation $\operatorname{Re}=U d_{1} / v=(1 \sim 3) \times 10^{4}(U$ : mean velocity of water flow, $v$ : water viscosity) and $\alpha_{\mathrm{v}}=0 \sim 10 \%$.

\section{Results and Discussion}

\subsection{Flow pattern}

Figure 3 illustrates the visualized flow pattern and schematic sketch, for the two-phase flow system $(R e=$ $2 \times 10^{4}, \alpha_{\mathrm{v}}=5 \%$ ) flowing into the contraction area when $A=0.18$. The white colored part in the picture denotes the gas (bubbles) since light is scattered due to the difference between the gas phase and liquid phase flexibility. The flow detaches from section B where the step is, and after the contraction step vortices are generated (hereafter, called vortex II), a gas pocket region is created with length approximately of $2.0 d_{2}$ and width of $0.12 d_{2}$. These vortices significantly affect the flow and cause pressure loss and flow instability.

The flow visualization and sketch for the same flow conditions, but when a ring at distance $L_{\mathrm{r}} / H=1$ is mounted before the contraction step is shown in Fig. 4. As the figure clearly shows, because of the ring effect, the vortices generated after the contraction (vortex II) are significantly suppressed. Also the gas pocket observable in the previous figure is greatly suppressed. Similar results were obtained in the case of single-phase flow by, observing the styrene

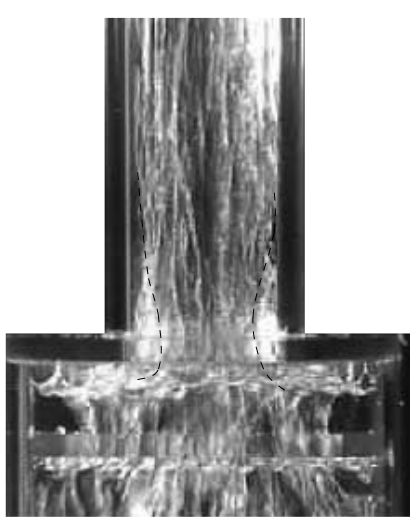

(a) Flow visualization

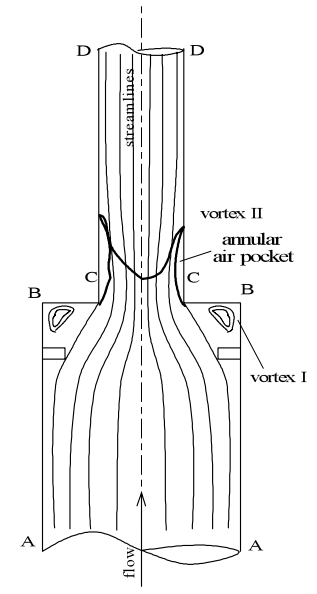

(b) Schematic flow diagram
Fig. 4 Flow pattern with ring, $L_{\mathrm{r}} / H=1$ $\left(R e=2 \times 10^{4}, \alpha_{\mathrm{v}}=5 \%, A=0.18\right)$

bead tracers, which showed a significant reduction of vortex II and the flow became smoother.

In general, when increasing the volumetric air fraction, the flow pattern changes from a bubbly state to an agitated bubbly state, which is disturbed bubbly state, and semi slug flow.

Downstream of the contraction bubble swarms and clusters with high and low bubble densities appear.

\subsection{Numerical analysis results}

As mentioned previously, an incompressible twofluid model with finite element method ${ }^{(6)}$ (FEM) was used. It is postulated that the gas and liquid phases are incompressible and that the mixture flows isothermally without any phase change. A one-pressure, two-fluid model provides the conservation equations of mass and momentum for each phase:

$$
\begin{aligned}
\frac{\partial \alpha_{k}}{\partial t}+\frac{\partial}{\partial x_{i}}\left(\alpha_{k} u_{k i}\right) & =0 \\
\frac{\partial u_{k i}}{\partial t}+u_{k i} \frac{\partial u_{k i}}{\partial x_{j}}= & -\frac{1}{\rho_{k}} \frac{\partial p}{\partial x_{i}}-\lambda_{k}\left(u_{g i}-u_{l i}\right) \\
& +\frac{1}{\rho} \frac{\partial \tau_{k i j}}{\partial x_{j}}+f_{k i}+g_{i}
\end{aligned}
$$

where

$$
\alpha_{g}+\alpha_{l}=1
$$

The subscript $k$ denotes the gas $(k=g)$ or liquid $(k=l)$ phase, and the subscripts $k, g$ and $l$ disobey the summation convention in this study. In Eq. (2), $\lambda_{k}$ is the interfacial friction coefficient, which is given as follows:

$$
\begin{aligned}
& \lambda_{g}=\frac{1}{8}\left(\alpha \rho_{1} C_{D} / \alpha_{g} \rho_{g}\right)\left|u_{g}-u_{l}\right| \\
& \lambda_{l}=-\frac{1}{8}\left(\alpha C_{D} / \alpha_{l}\right)\left|u_{g}-u_{l}\right|
\end{aligned}
$$

where $\alpha=6 \alpha_{g} / d_{p}$ and is given by the following equation considering the effect of bubble swarm: 


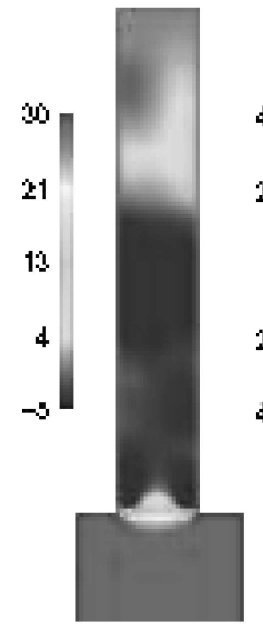

(a) Pressure

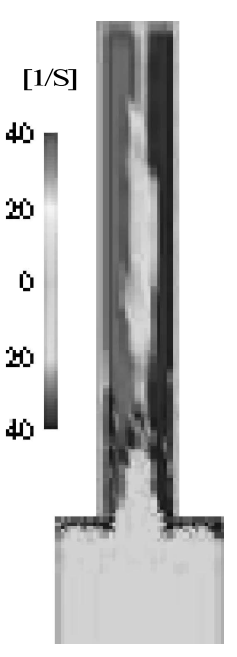

(b) Vorticity

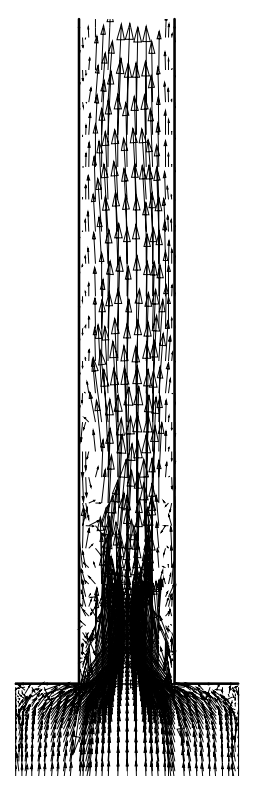

(c) Velocity vector
Fig. 5 Numerical analysis results, without ring $\left(\operatorname{Re}=2 \times 10^{4}, \alpha=10 \%, A=0.18\right)$

$$
C_{D}=\left[\frac{24}{\operatorname{Re}_{b}}\left(1+0.15 \operatorname{Re}_{b}^{0.687}\right)+\frac{0.42}{1+42500 \operatorname{Re}_{b}^{-1.16}}\right] \alpha_{l}^{-3.5}
$$

where, $R e_{b}=\rho_{l} d_{p}\left|u_{g}-u_{l}\right| / \mu_{l}$.

$\tau_{k}$ is the viscous stress, and $f_{k}$ is the interfacial forces (e.g., the virtual mass force and lift force) exclusive of the drag force.

In the present study it is postulated that a fully agitated two-phase mixture flows into the calculation domain in this calculation. In this case, there exists no slip velocity between the two-phases on the inlet section of the domain $C_{0}$ and both the volumetric fractions and the velocities distribute uniformly on the section. A traction free condition is assumed on the outlet boundary and a slip condition is prescribed on the lateral boundaries of the domain. At this present stage no turbulent model and diffusion models are employed for the gas phase in this calculation.

The entraining bubbles in the flow were postulated as spherical type with a diameter of $d_{b}=2.5 \mathrm{~mm}$. The time step for the calculating the single-phase flow was $5 \times 10^{-5}$ second, while for the case of two-phase flow it was $1 \times 10^{-6}$.

The flow conditions and the calculated width dimensions were the same as that in the experimental test section, while the length was shorter.

The results of pressure, vorticity and velocity vector for the case in which the ring is not inserted are shown in Fig. 5, when the area ratio is $A=0.18$ and the pressure is in a non-dimensional form using the kinetic energy $\rho_{\mathrm{w}} U^{2} / 2$ (where $\rho_{\mathrm{w}}$ is the water density). The flow, as seen, is significantly disturbed by the contraction step and vortices are generated after the contraction step. (a) Pressure

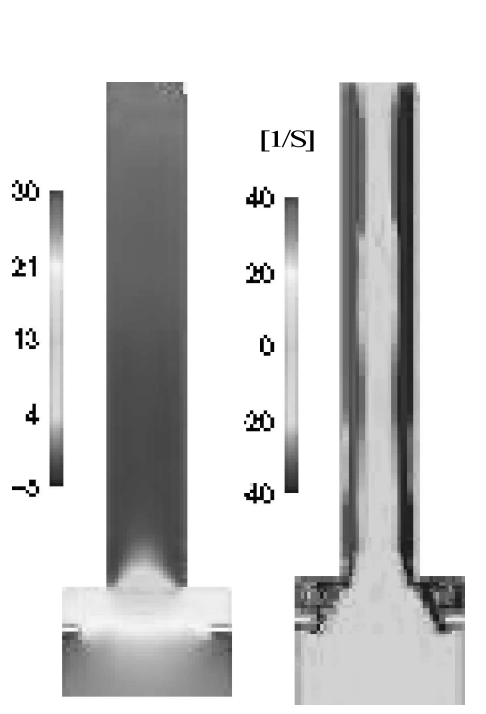

(b) Vorticity

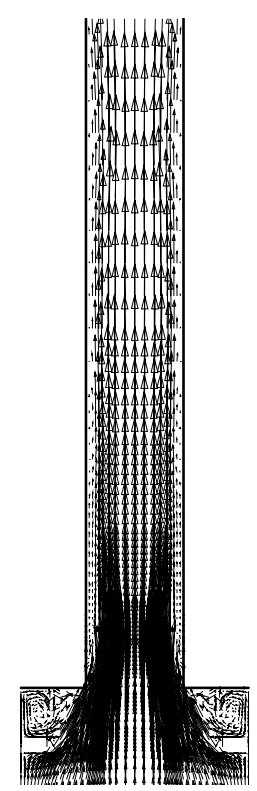

(c) Velocity vector
Fig. 6 Numerical analysis results, $L_{\mathrm{r}} / H=1$ $\left(\operatorname{Re}=2 \times 10^{4}, \alpha=10 \%, A=0.18\right)$

Figure 6 shows the results for the same flow condition but with a ring mounted at distance $L_{\mathrm{r}} / H=1$ before the step. Because of the ring, the flow is introduced more smoothly to the contraction area, as shown in the velocity vector, and a significant vortex decrease after the step is seen. Also, it is noted that the flow becomes stable again sooner than when the ring is not mounted. The flow pattern results from numerical analysis showed relatively good agreement compared to those taken from the visualization experiments.

\subsection{Pressure distribution}

The pressure distribution was measured experimentally using pressure holes along the pipe between the pressure $p_{1}$ at a reference point $x=0$ which is located at distance of $2 d_{1}$ upstream of the contraction and the pressure $p_{\mathrm{i}}$ taken at a distance of $x$ from the reference point. The pressure coefficient is given below:

$$
C_{\mathrm{p}}=2\left(p_{1}-p_{\mathrm{i}}-p_{\mathrm{e}}\right) /\left(\rho_{\mathrm{t}} U_{\mathrm{tm}}^{2}\right)
$$

where, $p_{\mathrm{e}}$ is the vertical elevation pressure $\left(=g \rho_{\mathrm{t}} H_{\mathrm{h}}, H_{\mathrm{h}}\right.$ : elevation between the $p_{1}$ and $\left.p_{\mathrm{i}}\right), \rho_{\mathrm{t}}$ is the two-phase mixture mean density $\left[=\rho_{\mathrm{w}}\left(100-\alpha_{\mathrm{v}}\right) / 100\right]$ and $U_{\mathrm{tm}}$ is the twophase mixture mean velocity $\left[=U_{\mathrm{m}}\left(100-\alpha_{v}\right) / 100\right]$.

Figure 7 illustrates the pressure distribution for $A=0.33$ and 0.18 respectively when the ring is not mounted and with a flow condition of $R e=1 \times 10^{4}$. The contraction step is located at distance $x / d_{1}=2$, while $x / d_{1}=0$ denotes the reference point. For the case of singlephase flow after the pressure is abruptly decreased due to the sudden contraction the flow is stabilized at an early stage $x / d_{1}>3$ (after the step) and it follows the pressure decrease gradient (Eq. of Blasius) caused by friction 


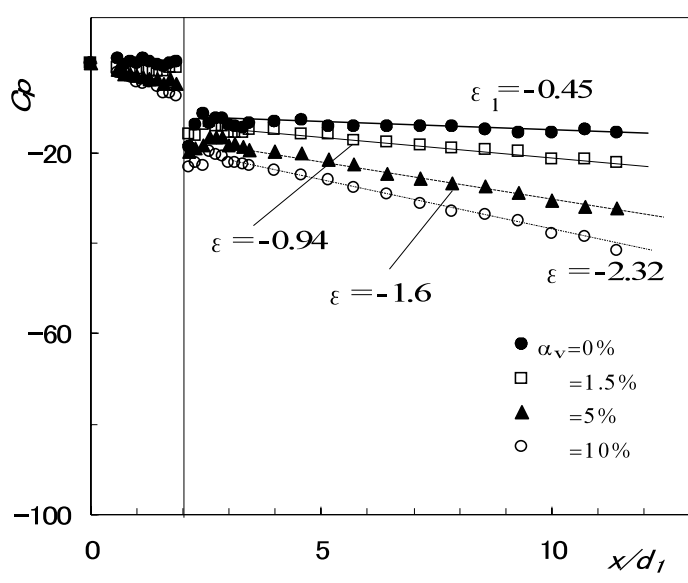

(a) $\quad A=0.33$

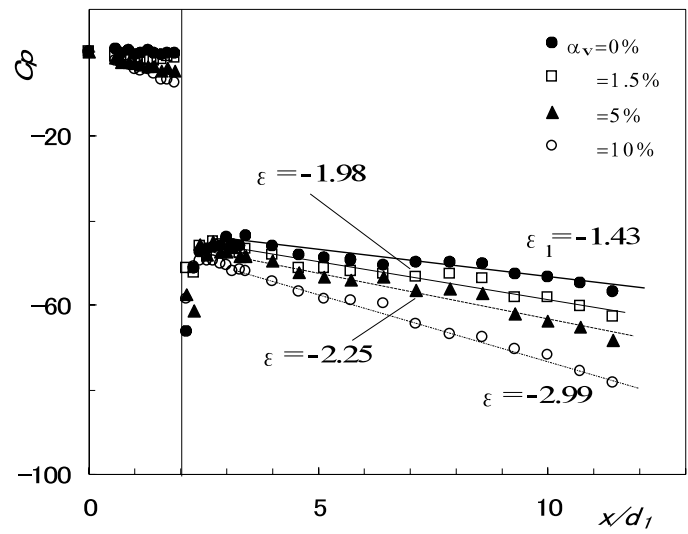

(b) $A=0.18$

Fig. 7 Pressure distribution without ring $\left(R e=1 \times 10^{4}\right.$, uncertainty in $\left.C_{\mathrm{p}} \pm 2\right)$

losses downstream. The equation gradient is given below:

$$
\varepsilon_{1}=\frac{\partial C_{p}}{\partial\left(x / d_{1}\right)}=-\lambda\left(d_{1} / d_{2}\right)^{5}
$$

where, $\lambda=0.3164\left[\left(d_{1} / d_{2}\right) R e\right]^{-1 / 4}$

The pressure gradient when the area ratio is $A=0.33$ was calculated from the graph as $\varepsilon=-0.43$ while the value when calculated from the equation mentioned above is $\varepsilon_{1}=-0.45$ as shown in Fig. 7 (a) and shows good agreement. Also in the case of $A=0.18$ there was good agreement between the experimental and theoretical results.

From the pressure distribution of both area ratios it is clear that increasing the air volume ratio $\alpha_{\mathrm{v}}$ increases the pressure gradient, which means there is a higher slope. An increased gradient indicates an increase in the frictional factor and can be explained by the fact that when gas is introduced and the flow becomes two-phase we have an increase in the turbulence level ${ }^{(1)}$.

Forces such as the friction between the flow and the pipe surface, interaction between bubbles and liquid gravitational and acceleration forces must be considered. For the case of $A=0.18$ [Fig. 7 (b)] the pressure drop is significantly larger because of the small area ratio, though similar results were obtained. In this case the pressure gradient

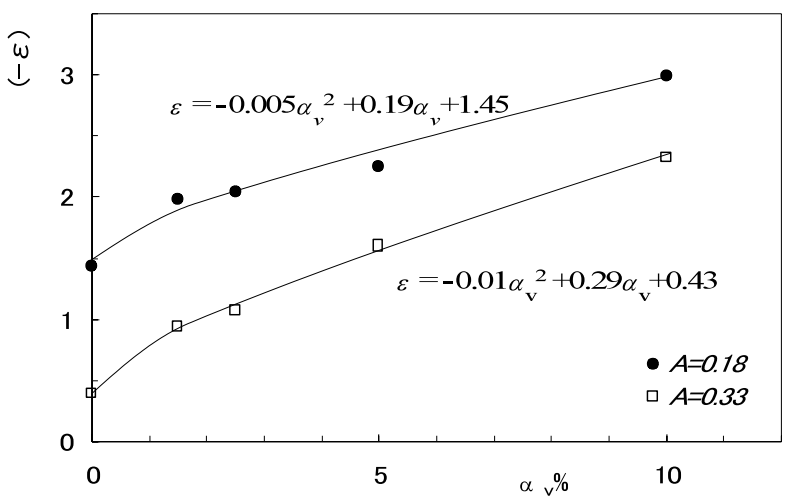

Fig. 8 Pressure gradient

$\left(R e=1 \times 10^{4}\right.$, uncertainty in $\left.\alpha_{\mathrm{v}} \pm 0.5\right)$

is also higher since the turbulence level is increased.

By mounting the ring at $L_{\mathrm{r}} / H=1 \sim 3$ before the contraction step similar results were seen, but the maximum pressure decrease, due to the sudden contraction, is reduced and the flow is stabilized at an earlier stage in both cases. This is explained in Fig. 4, taking into account the fact that when mounting an obstacle, the flow separates compulsorily and enters the contraction area more smoothly.

Figure 8 shows the results of the pressure gradient for both area ratios when $R e=1 \times 10^{4}$. It is clear that there is an almost linear relation to the pressure gradient increase by increasing the volumetric air fraction. Also, as Fig. 7 shows, increasing $\alpha_{\mathrm{v}}$ leads to an increase of $\varepsilon$, and can be expressed by $\alpha_{\mathrm{v}}$ as follows:

$$
\begin{aligned}
& \varepsilon=-0.05 \alpha_{\mathrm{v}}^{2}+0.29 \alpha_{\mathrm{v}}+0.43 \text { for } A=0.33 \\
& \varepsilon=-0.005 \alpha_{\mathrm{v}}^{2}+0.19 \alpha_{\mathrm{v}}+1.45 \text { for } A=0.18
\end{aligned}
$$

This is explained considering that the flow pattern after the contraction step depends on the air volume. Therefore, the increase of the velocity and gas void after the contraction is significantly different after the contraction depending on the area ratio and this significantly affects the results. From the visualization results it was observed that the flow varied from bubbly to agitated bubbly states and semi-slug with large bubble swarms created which explains the difference of the pressure gradient depending on $\alpha_{\mathrm{v}}$.

Thus, the contraction area ratio is in great dependence and a larger ratio $(A=0.33)$ denotes a smaller pressure gradient.

\section{4 Pressure drop at sudden contraction, $\Delta p$}

The pressure drop at the sudden contraction was obtained as follows from the pressure distribution shown in Fig. 7. The straight line that is drawn following the pressure distribution with a constant gradient before and after the contraction extends to the contraction position and the difference at the crossing points is the pressure drop $\Delta p$ due to contraction.

Figure 9 shows the pressure drop $\Delta p$ for $R e=1 \times 10^{4}$. 


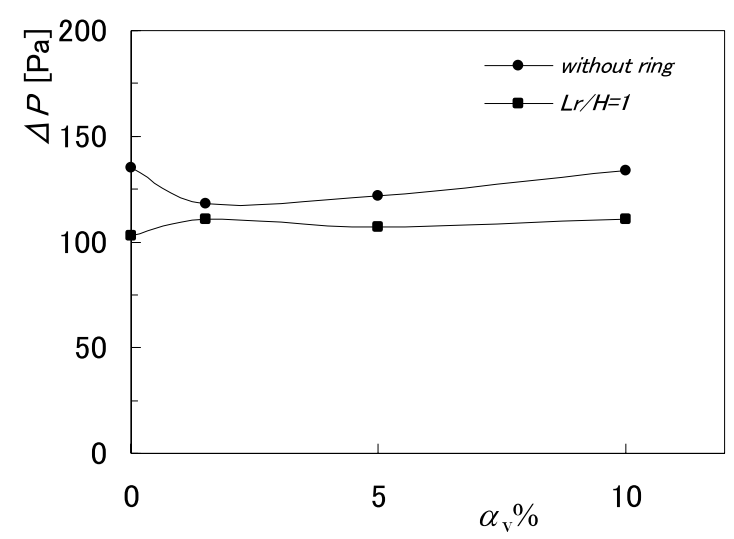

(a) $\quad A=0.33$

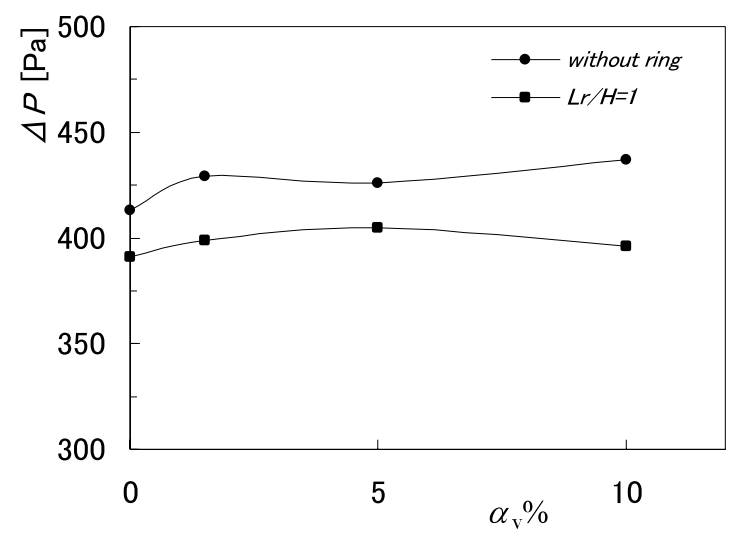

(b) $A=0.18$

Fig. 9 Pressure loss at sudden contraction $\left(R e=1 \times 10^{4}\right.$, uncertainty in $\left.\Delta p \pm 5 \mathrm{~Pa}\right)$

$\Delta p$ values for $A=0.18$ were considerably larger than the case of $A=0.33$. $\Delta p$ for single phase flow $(\alpha=0 \%)$ is in good agreement with previous results ${ }^{(8)}$. Figure 9 shows the pressure drop results in a case where the ring is mounted.

\subsection{Ring effect on pressure drop}

As shown in Fig. 9 when an obstacle is mounted at $L_{\mathrm{r}} / H=1$ the pressure drop decreases for every $\alpha_{\mathrm{v}}$. This depends on the flow that separates from the ring tip flowing along the contracted pipe and then the generated vortex is suppressed and controlled.

Figure 10 shows the drag reduction ratio $D_{\mathrm{R}}$. The ring in this case is a simple configuration that affects not only the single-phase flow case but also two-phase flow by reducing the drag at the sudden contraction. The drag reduction ratio for $A=0.33$ is larger than that for $A=0.18$.

\subsection{Flow fluctuation}

From the visualized flow pattern observation downstream of the contraction, there was an obvious change in the flow. Thick and thin bubble concentrations and bubble clusters were observed. In order to confirm the flow fluctuation, a semi-conductor type pressure transducer is installed at a distance of $2.5 d_{1}$ after the contraction and the pressure fluctuation following the flow fluctuation was

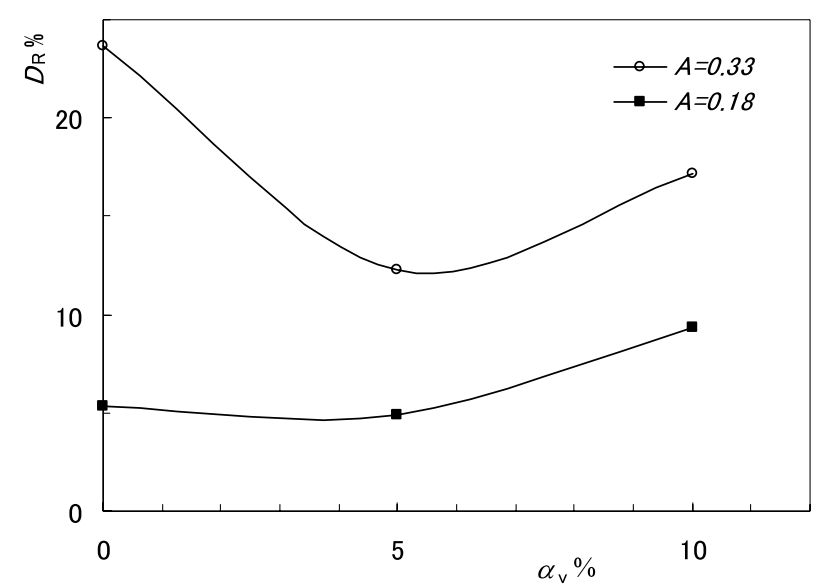

Fig. 10 Drag reduction rate, $L_{\mathrm{r}} / H=1$

$\left(R e=1 \times 10^{4}\right.$, uncertainty in $\left.D_{\mathrm{R}} \pm 0.5\right)$

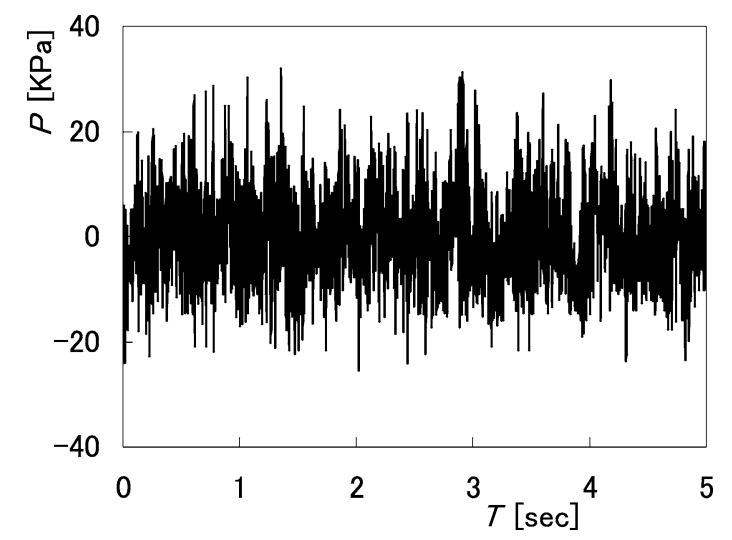

(a) Without ring

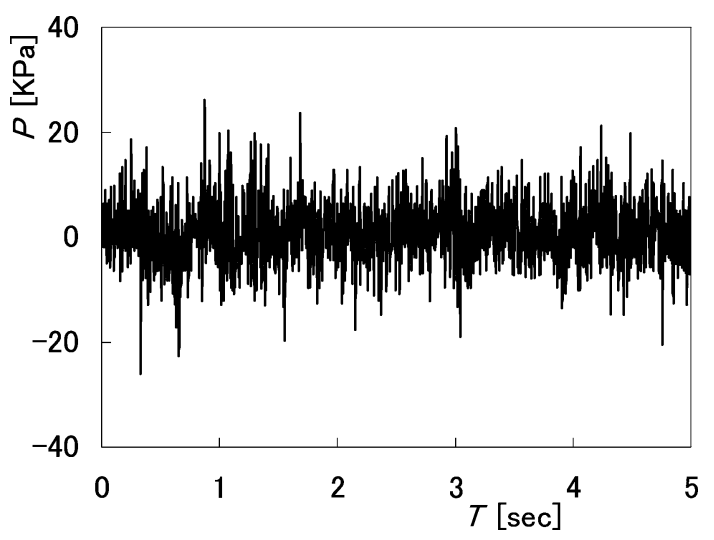

(b) $L_{\mathrm{r}} / H=1$

Fig. 11 Pressure fluctuation $\left(A=0.18, R e=2 \times 10^{4}, \alpha_{\mathrm{v}}=5 \%\right)$

measured.

Figure 11 shows the time series data of the pressure variation for $R e=2 \times 10^{4}$ and $\alpha_{\mathrm{v}}=5 \%$ when $A=0.18$. Figure 11 (a) shows a case without a ring. Multiple frequencies are combined and are justified from the existence of bubbles in the flow.

In Fig. 11(b), the case when ring is mounted at $L_{\mathrm{r}} / H=1$ for the same flow conditions is shown. The plot 


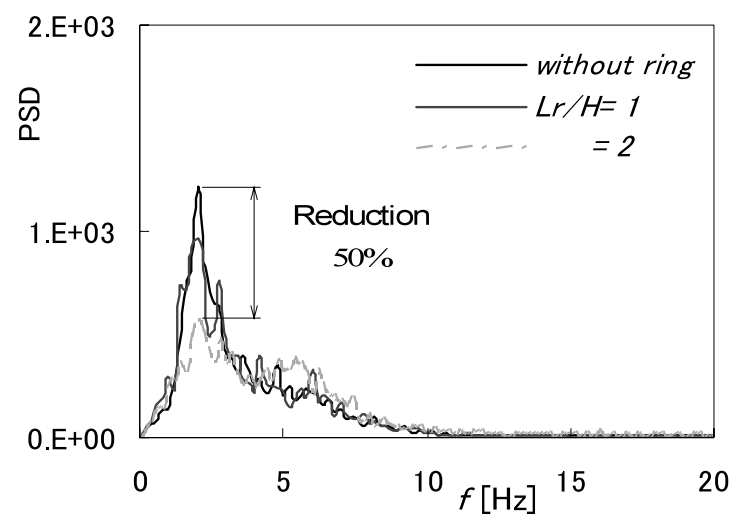

Fig. 12 PSD of pressure fluctuation $\left(A=0.33, \operatorname{Re}=2 \times 10^{4}, \alpha_{\mathrm{v}}=5 \%\right)$

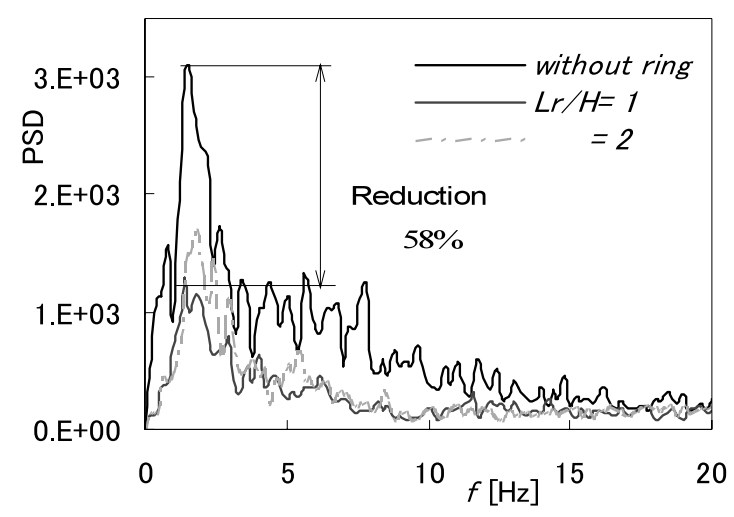

Fig. 13 PSD of pressure fluctuation $\left(A=0.18, R e=2 \times 10^{4}, \alpha_{\mathrm{v}}=5 \%\right)$

is almost similar though there is a clear difference in the amplitude, which has decreased. This is considered to be the effect of mounting an obstacle, in which the two-phase flow becomes more dispersed and stable.

Since it is difficult to analyze the pressure fluctuation data, FFT analysis was conducted for further investigation of the fluctuation properties.

Figure 12 illustrates the power spectrum density (PSD) with variety of ring position $L_{\mathrm{r}} / H=0 \sim 2$ while the flow condition is at $R e=2 \times 10^{4}$ and $\alpha_{\mathrm{v}}=5 \%$ with $A=0.33$. The ring effect is clearly shown in the PSD values, which have decreased remarkably. When ring was mounted at $L_{\mathrm{r}} / H=1$ the showed maximum decrease. The primary, dominant fluctuation had a frequency of approximately $f=2 \mathrm{~Hz}$.

Similarly, Fig. 13 shows the results for $A=0.18$ under the same flow conditions outlined previously and $f=1.6 \mathrm{~Hz}$. Likewise, a significant reduction in the fluctuation amplitude was confirmed which suggests the effect of the obstacle.

The $x$-axis spectra in Figs. 12 and 13 are reduced to values up to $20 \mathrm{~Hz}$ since the main interest in this study is to clarify whether the proposed method is effective mainly in terms of primary fluctuation. It has been determined that

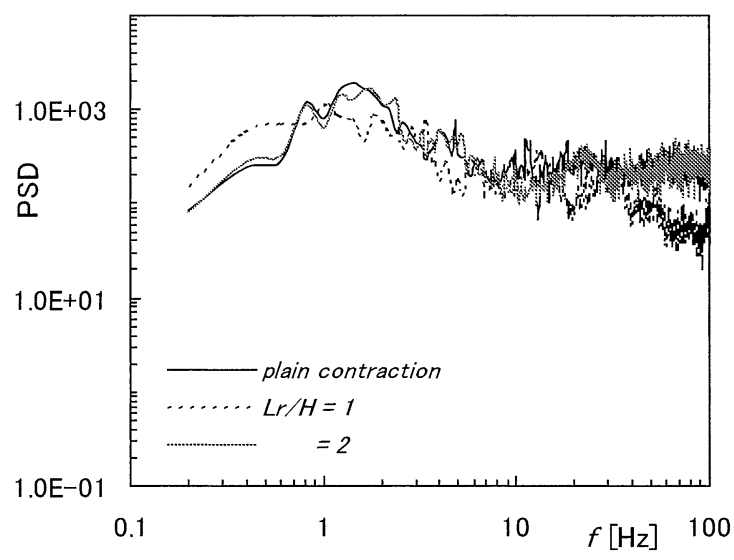

Fig. 14 PSD of pressure fluctuation $\left(A=0.18, R e=2 \times 10^{4}, \alpha_{\mathrm{v}}=10 \%\right)$

the range of primary fluctuation does not exceed the above mentioned value in any flow condition. Nonetheless, subfrequencies exist and the vibration for the whole range is illustrated in Fig. 14.

Mounting a ring forces the flow to enter the contraction more smoothly. Although the primary (maximum) frequency is almost the same, the fluctuating amplitude decreased in all flow conditions for both area ratios.

\section{Conclusion}

A study on the flow characteristics and flow control of a vertical upward two-phase flow was conducted when an abrupt contraction exists. From the experiments, flow visualization and numerical analysis conducted, the following main results are presented.

( 1 ) For single-phase flow (water) a large pressure drop occurs because of the contraction, and the flow becomes stable again at a relatively early position, where after $x / d_{1}>3$ the pressure gradient agreed well with that of the proposed equation (Eq. of Blasius), which is a result of wall friction.

(2) For the case of two-phase flow, a significant pressure decrease occurs. In general, increasing volumetric air fraction causes an increase in the pressure gradient, which is related to the turbulence level increase and to the change of the flow pattern from bubbly to agitated bubbly and semi-slug depending on the area ratio.

(3) Pressure loss at the sudden contraction is decreased by mounting a ring with its maximum at $23 \%$ for single phase for $A=0.33$.

(4) After the contraction, bubble swarms and clusters were formed and flow fluctuation occurred. Its dominant frequency varied depending on the area ratio. The frequency was $f=2$ and $1.6 \mathrm{~Hz}$ for $A=0.33$ and 0.18 respectively, but it did not change when the obstacle was mounted upstream the contraction.

( 5 ) Positive effect is confirmed with the vibration amplitude decreasing when mounting an obstacle (ring) 
before the contraction step in all flow conditions, varying $R e$ number, volumetric void fraction and area ratio. The best ring position in almost all conditions was at $L_{\mathrm{r}} / H=1$ with an achieved reduction rate up to $58 \%$ at its maximum.

\section{Acknowledgment}

The authors would like to thank Prof. T. Uchiyama, Nagoya Univ. for his valuable suggestion regarding numerical analysis.

\section{References}

(1) Cheremisinoff, N.P., Encyclopedia of Fluid Mechanics, Vol.3 (1985), pp.404-469, 563-606, 715-769.

( 2 ) Geiger, G.E., Sudden Contraction Losses in Single and Two-Phase Flow, Ph.D. Dissertation, Univ. of Pittsburgh, (1964).

( 3 ) Chiang, T.P. and Sheu, T.W.H., Bifurcations of Flow through Plane Symmetric Channel Contraction., Trans. ASME, Int. J. Heat Transfer, Vol.42 (1999), pp.3019-
3035.

(4) Ishii, M., Thermo and Fluid Dynamic Theory of TwoPhase Flow, (1975), Eyrolles, Paris.

( 5 ) Blevins, R.D., Applied Fluid Dynamics Handbook, Van Nostrand Reinhold Company Inc., (1984).

( 6 ) Uchiyama, T., Nuc. Eng. and Des., Vol.193 (1999), pp.145-157.

( 7 ) Ando, T., Shakouchi, T. and Miyata, K., Drag Reduction of Abrupt Contraction Pipe (Control of Flow Separation by Small Obstacle), Trans. Jpn. Soc. Mech. Eng., (in Japanese), Vol.70, No.691, B (2004), pp.650-656.

( 8 ) Kays, W.M., Loss Coefficients for Abrupt Changes in Flow Cross Section, Trans. ASME, Vol.72 (1950), pp.1067-1074.

( 9 ) Morimoto, Y., Madarame, H. and Okamoto, K., Fluctuation Phenomenon of Two-Phase Flow through Vertical Pipe with Contraction, Proc. of 3rd International Symposium on Two-Phase Flow Modeling and Experimentation, Pisa, Italy, (2004), CD-ROM. 